Introduction An estimated 35\% of cases of colorectal cancer (CRC) are due to heritable factors, and approximately $30 \%$ of the UK population has a family history of CRC. Those at hereditary risk should be effectively managed through registration, phenotypic and genotypic characterisation, and riskstratified colonoscopic surveillance. We assessed the impact of surveillance in patients at hereditary risk of CRC managed through the Family History of Bowel Cancer Registry at West Middlesex University Hospital (WMUH). Through analysis of this registry data, we assessed the diagnostic yield of colonoscopic surveillance and assessed the role of molecular testing.

Methods We analysed prospectively collected colonoscopic surveillance data in 361 patients undergoing surveillance at WMUH between 2010-2018. Patients were divided into five risk groups based on current BSG guidelines. Patient demographics including age, gender and family history were collated alongside colonoscopy findings and molecular data including mismatch repair (MMR) status. Impact of these variables on the prevalence of non-advanced adenomas (NAAs) and advanced adenomas (AAs) were assessed by logistic regression using SPSS software. Time to adenoma or AA was determined by survival analysis and findings were compared between index and surveillance colonoscopy.

Results In total, 640 colonoscopies were performed with 1000.1 years of follow-up. Five CRCs, 49 AAs and 170 NAAs were identified. The prevalence of CRC, AA and NAAs in patients without Lynch syndrome (LS) was $0.58 \%$, $8.19 \%$ and $26.02 \%$, respectively. Only age was significantly associated with both NAA and AA detection on multivariable analysis $(\mathrm{P}<0.05)$. Time to AA was earlier in LS patients (figure 1). A normal index colonoscopy was strongly associated with a normal finding during surveillance $(\mathrm{p}<0.001)$. In $38.6 \%$ of patients, molecular testing significantly altered surveillance strategies by recategorising familial risk.

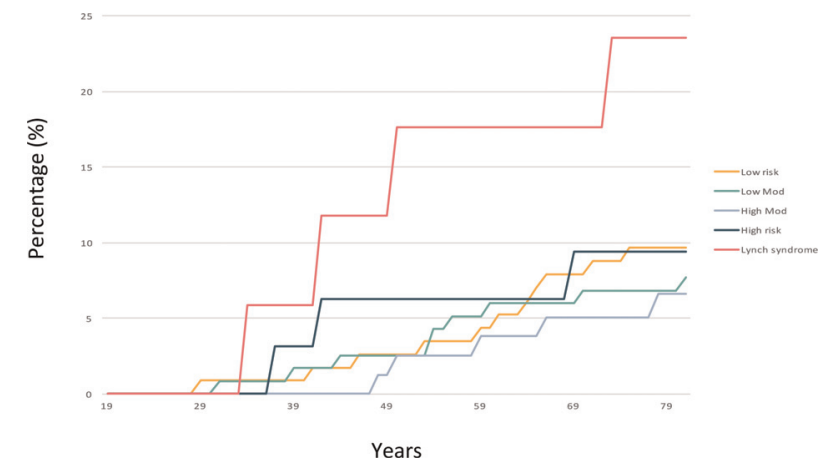

Abstract PTH-047 Figure 1 Cumulative proportion of AAs during colonoscopic surveillance according to age

Conclusion This data emphasises the strong association of colorectal neoplasia with MMR status and the need to exclude LS in patients at familial risk. Age is independently associated with colorectal neoplasia risk in this analysis, however patients often undergo colonoscopic surveillance inappropriately early. Finally, a normal index colonoscopy is associated with a low diagnostic yield in subsequent colonoscopies in this population. This low yield may influence future guideline strategies.

\section{PTH-048 COLONOSCOPIST KEY PERFORMANCE INDICATORS AND THE SURVEILLANCE OF PATIENTS WITH FAMILY HISTORY OF COLORECTAL CANCER}

${ }^{1}$ Benjamin Norton*, ${ }^{2}$ Thomas Wright, ${ }^{2}$ James Coultas, ${ }^{2}$ Victoria Nicholas,

${ }^{1,2}$ Kevin Monahan. ${ }^{1}$ Genomic Medicine, Imperial College London, London, UK; ${ }^{2}$ Department of Gastroenterology, West Middlesex University Hospital, Chelsea and Westminster Hospital NHS Trust, London, UK

\subsection{6/gutjnl-2019-BSGAbstracts.73}

Introduction Colorectal cancer (CRC) accounts for over 40,000 new cases/year in the UK, and 35\% of cases develop due to inherited susceptibility. Patients at hereditary risk undergo colonoscopic surveillance to reduce CRC incidence and mortality. High quality colonoscopy, measured by key performance indicators (KPI) including adenoma detection rate (ADR), caecal intubation rate (CIR), and polyp recovery rate (PRR) may enhance surveillance outcomes. This is important for high risk patients where colonoscopy may be a one-off intervention, or where there is significant risk of interval cancers. Consequently, we assessed colonoscopy quality in a cohort at hereditary risk of CRC managed at West Middlesex University Hospital (WMUH), and determined their relationship to colonoscopist KPIs in non-surveillance cohorts.

Methods We analysed prospective colonoscopic surveillance data through the Family History of Bowel Cancer Registry at WMUH between 2010-19. A cohort of 361 patients at hereditary risk were divided into five risk groups based on current BSG guidelines. Patient demographics including age, gender and family history were collated alongside colonoscopist data, colonoscopy findings, and KPIs. Using linear regression (SPSS software), we compared findings to ADRs, CIRs and PRRs of colonoscopists in non-surveillance patients.

Results During surveillance, 640 colonoscopies were performed by 12 clinicians. The collective ADR during index, 1st, 2nd and 3 rd surveillance colonoscopies was 18.9\%, 16.0\%, 29.6\% and $25.8 \%$, respectively. There was no difference in ADRs at Index, 1st and 2nd surveillance colonoscopy between familial risk groups. The average ADR for colonoscopists across all surveillance colonoscopies was 22.6\% (8.3-35.7\%) and CIR was $97.92 \%(50-100 \%)$. In the non-surveillance cohort ADR was 29.5\% (13.3-40.7\%), CIR 93.5\% (78.8-95.7\%) and PRR 95.2\% (86.9-98.2\%). Colonoscopist ADRs during surveillance correlated with both ADRs $\left(\mathrm{R}^{2} 0.48, \mathrm{P}<0.05\right.$; figure 1$)$ and PRRs $\left(\mathrm{R}^{2} 0.47, \mathrm{P}<0.05\right)$ in the non-surveillance cohort but not CIR.

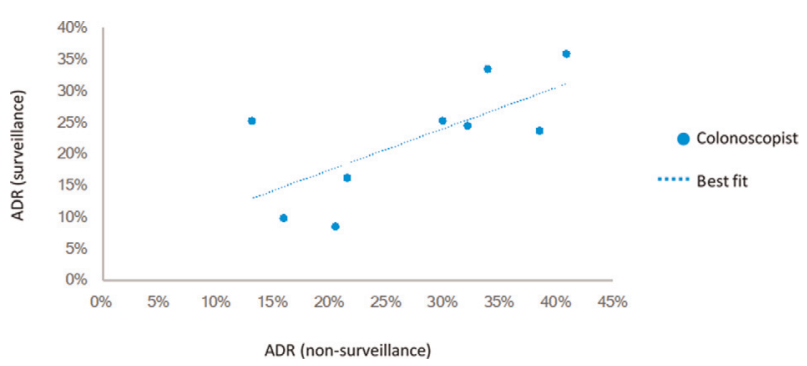

Abstract PTH-048 Figure 1 Colonoscopist ADRs in surveillance \& non-surveillance

Conclusion ADR is the main indicator of efficacy during colonoscopy and a consistent independent risk factor for interval CRCs in screening colonoscopies. Colonoscopists ADRs in 
patients undergoing surveillance correlated with their ADRs and PRRs during colonoscopies in a non-surveillance cohort. Therefore, we suggest that all surveillance colonoscopies are performed by endoscopists consistently achieving BSG colonoscopy KPI minimum standards.

\section{PTH-049 AUDIT OF 27 YEARS OF ENDOSCOPY PRACTICE AT THE UNIVERSITY COLLEGE HOSPITAL, IBADAN, NIGERIA}

Samuel Olawale Ola*, Kolawole Oluseyi Akande, Christopher B Summerton. University College Hospital, Ibadan Nigeria, Ibadan, Nigeria

\subsection{6/gutjil-2019-BSGAbstracts.74}

Introduction Endoscopy is an important and useful invasive procedure in patient management and it is rapidly evolving requiring regular update. Hence the need to audit the endoscopic practice in a low economic clime such as Nigeria to ensuring standardization of the procedure towards better patient management, training and research.

Methods The study was a retrospective analysis of the record of endoscopic procedures carried out the Endoscopy Suites of Nigeria's premiere University College Hospital, Ibadan from 1992 to 2018. It involved a review of the biodata of the patients, types of endoscopy procedures carried out versus patient population, experience of the mentor and mentee endoscopists, availability of equipment for endoscopy, leakage testing and therapy, disinfection, storage, report software and other resources towards patient care.

Results From table 1, a total of 7050 patients underwent endoscopy between 1992 and 2018 with 71.4\% of the subjects had GIT procedures. Although GIT endoscopy started in 1978, it was not until 1992 that a record of the procedures was commenced with 4 procedures carried out in 1992 which has grown exponentially yearly to 715 procedures in 2018. Only one consultant GIT endoscopist was available in 1992 and this increased to 8 in 2018. The Endoscopy practice also had 7 Consultant Bronchoscopists and 8 Laryngoscopists at 2018 although the practice has trained 78 trainees with less than 10 endoscopies during training but gained further experience during consultancy practice.

The male female ratio of all the patients for the 7050 procedures (9 Paediatric patients) from 1992 to 2018 is 6:5 with mean age of $50.3 \pm 17.7$ years and age range of 5 to 88 years. The predominant age group of all patients was 40-50 years age group accounting for $15.5 \%$. The procedures were mostly diagnostic with $47.5 \%$ of the patients for upper GIT studies. It is of saddened note that bronchoscopy service has stopped in the 2018 principally due to ageing bronchoscope. In spite of the limited and basic equipment available, the centre ran two endoscopic - pathology workshops in 2007 and 2014 with 50 nurses and 40 doctors at attendance. With the centre serving the whole West Africa subregion, the centre lacked endoscopy report system, facilities for therapeutic endosccopy including ERCP and endoscopy ultrasonography.

Conclusions Although, the centre had been carrying out endoscopy procedures over three decades, this has been predominantly diagnostic endoscopy with limited trainee exposure. Hence there is a need for standardization endoscopy practice amidst other requirements for uplift in endoscopy services, training and research to augment patient care.

Abstract PTH-049 Table 1 Pattern of procedures carried out on patients for Endoscopy at University College Hospital, Ibadan; Nigeria

\begin{tabular}{|c|c|c|c|c|c|}
\hline Procedures & 1992-2017 & 2018 & total & & $\%$ \\
\hline Diagnostic & 2900 & 448 & 3348 & & 47.5 \\
\hline Upper GIT & 1201 & 252 & 1453 & & 20.6 \\
\hline Colonoscopy & 206 & 15 & 221 & & 3.1 \\
\hline Sigmoidoscopy & 2 & 0 & 2 & & $2.8 \times 10^{-4}$ \\
\hline ERCP & 8 & 0 & 8 & & 0.1 \\
\hline Proctoscopy & 4317 & 715 & 5032 & & 71.4 \\
\hline \multicolumn{6}{|l|}{ Subtotal } \\
\hline Laryngoscopy & 750 & 42 & 792 & & 11.2 \\
\hline Bronchoscopy & 89 & 0 & 89 & & 1.3 \\
\hline Subtotal & 5156 & 757 & 5913 & & 83.9 \\
\hline No record & 1137 & 0 & 1137 & & 16.1 \\
\hline Total & 6197 & 757 & 7050 & & 100 \\
\hline \multirow[t]{2}{*}{ Therapeutic } & Variceal banding & Polypectomy & & PEG & \\
\hline & 16 & 1 & & 1 & \\
\hline No of procedures & GIT & Bronchoscopy 3 & & ENT & \\
\hline Mentors $>300$ & 8 & 4 & & 2 & \\
\hline$<110$ & 3 & 4 & & 7 & \\
\hline Mentees < 10 & 19 & & & 55 & \\
\hline Equipment Present & \multicolumn{5}{|c|}{ Leakage tester, Manual disinfection, Urea Breath test kit, Heater probe, Video-endoscopy with HDI, Vital Scopes } \\
\hline Year & $1992-2000$ & $2001-2010$ & $2011-2$ & & \\
\hline No of patients & 211 & 2555 & 428 & & \\
\hline Ratio & 1 & 12 & 20 & & \\
\hline
\end{tabular}

